\section{T6 UNDERSTANDING THE CELLULAR DYSFUNCTION CAUSED BY PATHOGENIC SURFACTANT PROTEIN C MUTANT I73T}

JA Dickens, MO Ellis, SJ Marciniak. University of Cambridge, Cambridge, UK

\subsection{6/thoraxjnl-2017-210983.6}

Introduction and Objectives Our understanding of the pathogenesis of interstitial pulmonary disease is limited largely to its late, fibrotic stages. Inherited forms offer the potential to understand earlier pathogenic events. Autosomal dominant mutations in surfactant protein C (SFTPC) cause some cases of familial pulmonary fibrosis; the most common mutation is I73T, which is said to be mistrafficked within the type 2 pneumocyte. We hypothesise that mistrafficking of SFTPC I73T is causal in triggering type 2 pneumocyte dysfunction in familial pulmonary fibrosis. This work aims to understand the mechanism of SFTPC I73T mistrafficking such that type 2 pneumocyte dysfunction in familial, and sporadic disease can be better understood.

Methods We overexpressed GFP tagged wild-type (WT) and I73T SFTPC in HeLa cells to study their localisation and trafficking. Subcellular localisation and co-localisation with organelle markers was assessed using confocal microscopy and immunofluorescence. Western blotting was used to assess expression of SFTPC isoforms and GFP traps to study SFTPC interactors, including ubiquitin. Mass spectrometry was used to assess the interactome of WT and I73T SFTPC.

Results In HeLa cells overexpressing GFP-SFTPC, we observed mistrafficking of SFTPC I73T; WT SFTPC is trafficked to the multivesicular body (MVB), while I73T mistrafficks to the cell surface and into tubular structures (figure 1A) which co-localise with a marker of recycling endosomes Rab8 (figure 1B). Correct trafficking of WT SFTPC to the MVB depends on ubiquitination of lysine-6 (K6). We see an absence of ubiquitination in the I73T mutant and a subcellular distribution similar to that seen in a SFTPC mutant (K6R) which cannot be ubiquitinated. Mass spectrometry revealed differential binding between WT and I73T SFTPC of a number of cell surface proteins.

Conclusion We have shown that pathogenic SFTPC mutant I73T mistrafficks not only to the cell surface, but also into recycling endosomes. We propose that this occurs as a result of failure of ubiquitination and internalisation into the MVB. Trafficking defects appear to impact upon binding of WT vs I73T to cell surface markers, the functional significance of which is the focus of our ongoing work.
A
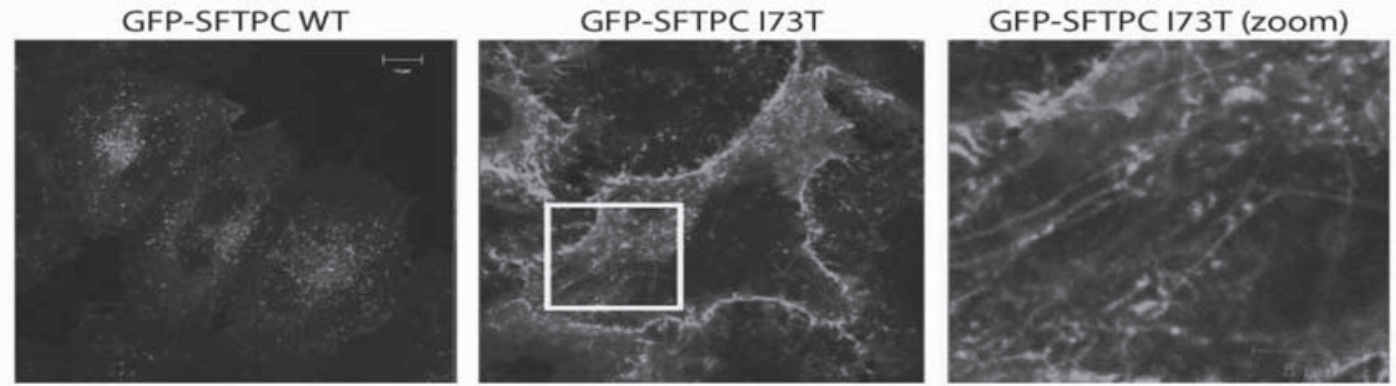

B

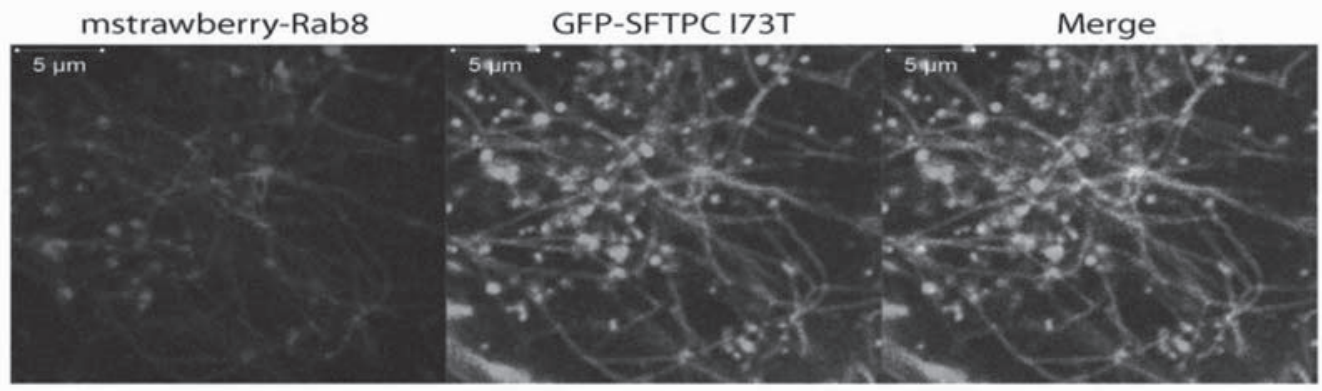

Abstract T6 Figure 1 SFTPC I73T localises to the cell surface and recycling endosomes. (A) Subceller localisation of GFP-SFTPC WT and I73T expressed in HeLa cells. (B) GFP-SFTPC co-localises with mstrawberry Rab8, a marker of recycling endosomes. 


\section{COPD: doing what works}

\section{S1 A 20 YEAR EXPERIENCE OF STAGED BILATERAL LUNG VOLUME REDUCTION FOR EMPHYSEMA - THE LONG TERM BENEFITS OF COMBINED SURGICAL AND ENDOBRONCHIAL TECHNIQUES}

${ }^{1}$ IF Oey, ${ }^{1} \mathrm{MC}$ Steiner, ${ }^{1} \mathrm{MDL}$ Morgan, ${ }^{2} \mathrm{DA}$ Waller. ${ }^{1}$ University Hospitals Leicester, Leicester, UK; ${ }^{2}$ Barts Health NHS Trust, London, UK

\subsection{6/thoraxjnl-2017-210983.7}

We made a decision early in our lung volume reduction (LVR) experience to offer a staged bilateral approach with the timing of each additional intervention determined by patient choice and have latterly incorporated the use of endobronchial valves into this protocol. We have analysed the long-term effect on patient outcome of this novel approach. Over a 20 year period 329 LVR procedures were performed on 256 patients (157 male, 99 female, median age 61 [23-79] years) by a single surgeon in one institution. Baseline lung function showed predicted values (mean +/-SE): FEV1 28 (11)\%, RV 253 (53)\%, DLCO 39 (39)\%. 64 patients have received a second LVR and 13 a third LVR procedure (fig). Median time between first and second stage was $3.8(0.1-12.5)$ years. The time interval between $2 \mathrm{nd}$ and $3 \mathrm{rd}$ stage was 2.7 (0.2-5.2) years. Median time interval between 1 st and 3rd stage was 5.8 (1.9-10) years. Overall 30 day mortality was $3 \%(20 \%$ after open, 3\% after VATS and 3\% after EBV). Median overall survival was 5.6 (95\% CI 4.7-6.9) years. In the subgroup of patients who underwent staged procedures there was a significant improvement in mean predicted FEV1 from $28 \%$ at initial baseline to $34 \%$ at 6 years. There was a sustained reduction in static lung volumes up to 8 years: predicted TLC remained reduced from $143 \%$ to $123 \%$ and predicted RV from $259 \%$ to $189 \%$. The physiological improvements after first and second stage were similar. There were also sustained improvements over initial baseline in health status as assessed by Euroquol for 5 years [50 $(+/-26)$ to $62(+/-23), \mathrm{p}<0.001]$ en in SF36: perception of the physical role was significantly improved for up to 7 years, social functioning for 8 years. Energy levels remained significantly higher for 9 years with $20 \%$ of patients scoring better than preoperatively. A staged unilateral video assisted surgical and endobronchial approach to lung volume reduction dictated by the patient is justified by a sustained benefit for up to 9 years in physiology and health status in patients with severe emphysema who had already received maximal medical therapy.

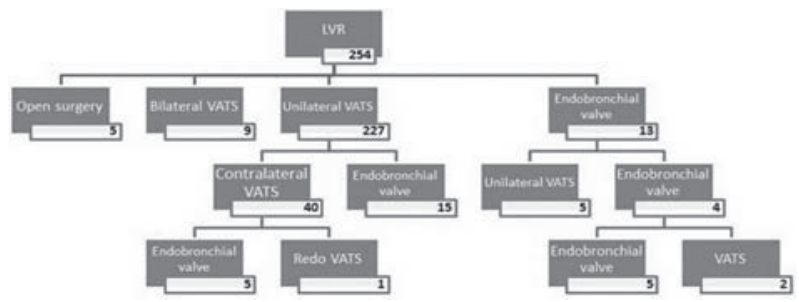

Abstract S1 Figure 1 All LVR procedures.

\section{S2 THE IMPACT OF PULMONARY REHABILITATION (PR) COMPLETION AND OUTCOME ON SUBSEQUENT HOSPITAL ADMISSIONS IN PATIENTS WITH COPD. RESULTS FROM THE NATIONAL COPD AUDIT}

MC Steiner, J Holzhauer-Barrie, D Lowe, V McMillan, V van Loo, CM Roberts. Royal College of Physicians, London, UK

\subsection{6/thoraxjnl-2017-210983.8}

Introduction Clinical trials suggest attendance at PR reduces subsequent hospitalisation rates. However, whether this benefit occurs in routine clinical practice is uncertain. We analysed admission rates and days spent in hospital 90 and 180 days after assessment for PR in the 2015 national PR audit cohort. Methods PR services across England and Wales provided data for all consenting patients assessed for PR between Jan and April 2015. Cumulative rates of admission to hospital and numbers of days spent in hospital at each timepoint (and between the 90 and 180 day timepoints) were extracted from Hospital Episode Statistics (HES) and compared between patients who did and did not complete PR and between those reaching and not reaching accepted thresholds for clinically important improvements (MCIDs) in exercise and health status measures.

Results Data for 7135 patients included in the audit in England were extracted. Overall admission rates (proportion with at least one admission) were $18.6 \%$ and $29.6 \%$ at 90 and 180 days respectively. At each timepoint, completion of PR (defined by there being a discharge assessment) was associated with a reduced risk of admission to hospital (compared with non-completion) (90 days: 13.1 vs $26.4 \%$; 180 days: 23.7 vs 37.9\%; 91-180 days: 15.0 vs $21.7 \%$; all $\mathrm{p}<0.001)$ and reduced days spent in hospital (90 days: Mean 3.1 vs 7.1 days; 180 days: mean 4.8 vs 9.4 days; 91-180 days: 4.91 vs 7.6 days; all $\mathrm{p}<0.001)$. Reaching the MCID for exercise measures after completion of PR was associated with reduced risk of admission at 180 days (and 91-180 days) $(\mathrm{p}<0.05)$ but not reduced days spent in hospital. Changes in health status measures were not associated with admission rates or days spent in hospital.

Conclusion Successful completion of PR is associated with reduced risk of hospitalisation and reduced days in hospital up to 180 days after assessment. Although some of this effect may be due to case-mix variation, actions to enhance uptake and completion rates for PR may be effective measures to reduce health care resource utilisation for patients with COPD.

\section{S3 CLINICAL CULTURES AND THE SENSATION OF BREATHLESSNESS}

J Macnaughton, R Oxley, A Rose. Durham University, Durham, UK

10.1136/thoraxjnl-2017-210983.9

Introduction and Objectives Our Wellcome Trust funded project, the Life of Breath, takes as its starting point the problem of symptom discordance between clinically measured lung function and patient experience. Clinicians have long recognised the influence of thoughts and emotions on symptom of breathlessness. Recent work in neuroscience, exemplified in the 'multidimensional model' of breathlessness proposed by 